\title{
Simplified Stability Criteria for Delayed Neutral Systems
}

\author{
Xinghua Zhang, ${ }^{1,2}$ Xiangyu Gao, ${ }^{1}$ and $\mathrm{Min} \mathrm{Su}^{1}$ \\ ${ }^{1}$ School of Mathematical Science, Heilongjiang University, Harbin 150080, China \\ ${ }^{2}$ College of Computer and Information Engineering, Heilongjiang University of Science and Technology, Harbin 150022, China
}

Correspondence should be addressed to Min Su; sumin_12345@sina.com

Received 4 March 2014; Accepted 1 April 2014; Published 13 May 2014

Academic Editor: Yuxin Zhao

Copyright (C) 2014 Xinghua Zhang et al. This is an open access article distributed under the Creative Commons Attribution License, which permits unrestricted use, distribution, and reproduction in any medium, provided the original work is properly cited.

\begin{abstract}
For a class of linear time-invariant neutral systems with neutral and discrete constant delays, several existing asymptotic stability criteria in the form of linear matrix inequalities (LMIs) are simplified by using matrix analysis techniques. Compared with the original stability criteria, the simplified ones include fewer LMI variables, which can obviously reduce computational complexity. Simultaneously, it is theoretically shown that the simplified stability criteria and original ones are equivalent; that is, they have the same conservativeness. Finally, a numerical example is employed to verify the theoretic results investigated in this paper.
\end{abstract}

\section{Introduction}

Some practical systems, such as population ecology, neural networks, heat exchangers, and robots in contract with rigid environments, have been modeled by functional differential equations of neutral type (e.g., [1-6]). Since stability analysis is the primary task of analyzing and synthesizing a system, many researchers have paid more and more attention to establish stability criteria for delayed neutral systems (see $[7,8]$ and the references therein).

For a class of linear time-invariant neutral systems with neutral and discrete constant delays, both neutral- and discrete-delay-dependent stability criteria have been investigated in [9-27]. Many approaches have been provided for obtaining these stability criteria. Here we mention only some critical and representative approaches. Model transformation approach first transforms the neutral system with discrete delay into the one with distributed delays, and then delaydependent stability criteria can be obtained by constructing a Lyapunov-Krasovskii functional [9]. However, model transformation approach introduces some additional dynamics, which leads still to conservative results [28]. To overcome this shortage, the so-called descriptor model transformation approach is introduced in $[15,24]$, which transforms the original system to an equivalent descriptor form. The descriptor model transformation approach will not introduce additional dynamics in the sense defined in [28]. Although the results obtained from descriptor model transformation approach may be less conservative than some existing ones, they can be improved by employing some other approaches (e.g., free weighting matrix approach $[21,22]$ and augmented Lyapunov-Krasovskii functional approach [14, 23, 27]) to get a larger bound for discrete delay.

By employing free weighting matrices and the LeibnizNewton formula, He et al. [21] presented a neutral- and discrete-delay-dependent stability criterion for neutral systems with neutral and discrete constant delays, which reduces the conservativeness of methods involving a fixed model transformation. For the same system models, Qian et al. [27] established neutral- and discrete-delay-dependent stability criteria by constructing a Lyapunov-Krasovskii functional with additional functional parameters and employing free weighting matrices. To the best of our knowledge, the large-scale employment of functional parameters and/or free weighting matrices can cause complex stability criteria, and thereby the computational complexity increases obviously. Therefore, in order to reduce computational complexity, it is necessary to reduce the number of functional parameters and free weighting matrices. This motivates the present study.

The aim of this paper is to simplify several stability criteria proposed by $\mathrm{He}$ et al. [21] and Qian et al. [27]. The main contributions of the paper are as follows. (i) In the premise of not increasing conservativeness, a pair of stability criteria proposed in [27, Theorem 1 and Corollary 1] are 
simplified by using matrix analysis techniques; (ii) one of the pair of simplified stability criteria is theoretically presented to be less conservative than [26, Corollary 1], and another is theoretically proven to be equivalent to [21, Theorem 1]; and (iii) by one numerical example, our theoretic results are verified to be more effective than many existing results.

\section{Problem Statement and Preliminary Results}

Consider a class of delayed neutral systems described as

$$
\begin{gathered}
\dot{x}(t)-C \dot{x}(t-\eta)=A x(t)+A_{1} x(t-\tau), \quad t \geq 0, \\
x(t)=\phi(t), \quad t \in[-\omega, 0],
\end{gathered}
$$

where $x(t)$ is the $n$-dimensional state vector, $A, A_{1}$, and $C$ are known real constant matrices with appropriate dimensions, $\omega=\max \{\eta, \tau\}, \phi(s)$ is an $\mathbb{R}^{n}$-valued continuous initial function defined on $[-\omega, 0]$, and positive scalars $\eta$ and $\tau$ represent the neutral and discrete delays, respectively.

Define $C\left([-\eta, 0], \mathbb{R}^{n}\right)$ as the set of continuous $\mathbb{R}^{n}$ valued function on the interval $[-\eta, 0]$, and let $x_{t} \in C\left([-\eta, 0], \mathbb{R}^{n}\right)$ be a segment of system trajectory defined as

$$
x_{t}(\theta)=x(t+\theta), \quad \theta \in[-\eta, 0] .
$$

Define an operator $\mathscr{D}: C\left([-\eta, 0], \mathbb{R}^{n}\right) \rightarrow \mathbb{R}^{n}$ by $\mathscr{D} x_{t}=$ $x(t)-C x(t-\eta)$. The definitions on stability of operator $\mathscr{D}$ and systems (1a) and (1b) can be seen in [29].

As mentioned in Introduction, neutral- and discretedelay-dependent stability criteria for systems (1a) and (1b) have been investigated by $\mathrm{He}$ et al. [21] and Qian et al. [27]. In this paper, we will simplify the stability criteria [21, Theorem 1] and [27, Theorem 1 and Corollary 1] in the premise of not increasing conservativeness of stability criteria.

In order to present conveniently our main results, the following lemmas are required.

Lemma 1 (Schur complement lemma [30]). Given constant matrices $\Omega_{1}, \Omega_{2}$, and $\Omega_{3}$ of appropriate dimensions, where $\Omega_{1}^{T}=\Omega_{1}$ and $\Omega_{2}^{T}=\Omega_{2}$, then

$$
\Omega_{1}+\Omega_{3}^{T} \Omega_{2}^{-1} \Omega_{3}<0
$$

if and only if

$$
\left[\begin{array}{cc}
\Omega_{1} & \Omega_{3}^{T} \\
\Omega_{3} & -\Omega_{2}
\end{array}\right]<0 \quad \text { or }\left[\begin{array}{cc}
-\Omega_{2} & \Omega_{3} \\
\Omega_{3}^{T} & \Omega_{1}
\end{array}\right]<0 .
$$

Lemma 2 (see [31]). Given a real symmetric matrix $\Psi$ and a pair of real matrices $P$ and $Q$, the following $L M I$ problem

$$
\Psi+P^{T} X^{T} Q+Q^{T} X P<0
$$

is solvable with respect to decision variable $X$ if and only if

$$
\mathcal{N}_{P}^{T} \Psi \mathcal{N}_{P}<0, \quad \mathcal{N}_{\mathrm{Q}}^{T} \Psi \mathcal{N}_{\mathrm{Q}}<0,
$$

where $\mathcal{N}_{P}$ and $\mathcal{N}_{\mathrm{Q}}$ are matrices whose columns form a basis of the right null spaces of $P$ and $Q$, respectively.

\section{Several Stability Criteria in the Literature}

In this section we introduce several existing stability criteria for systems (1a) and (1b), which will be useful to present conveniently the main results of this paper in the next section.

Proposition 3 (see [21, Theorem 1]). For given scalars $\tau>0$ and $\eta>0$, systems (1a) and (1b) are asymptotically stable, if the operator $\mathscr{D}$ is stable and there exist real $n \times n$ matrices $P^{T}=$ $P>0, Q_{i}^{T}=Q_{i}>0(i=1,2), R^{T}=R>0, \check{X}_{i i}^{T}=\check{X}_{i i}$, $\check{Y}_{i i}=\check{Y}_{i i}(i=1, \ldots, 5), \check{X}_{i j}$, and $\check{Y}_{i j}(1 \leq i<j \leq 5)$ such that the following LMIs are feasible:

$$
\begin{gathered}
{\left[\begin{array}{cc}
\check{X} & \check{X}_{5}^{T} \\
\check{X}_{5} & \check{X}_{55}
\end{array}\right] \geq 0, \quad\left[\begin{array}{cc}
\check{Y} & \check{Y}_{5}^{T} \\
\check{Y}_{5} & \check{Y}_{55}
\end{array}\right] \geq 0,} \\
{\left[\begin{array}{cc}
\check{\Phi}+\tau \check{X}+\eta \check{Y}+\check{\Phi}_{X}+\check{\Phi}_{Y} & \check{A}^{T} S \\
S \check{A} & -S
\end{array}\right]<0,}
\end{gathered}
$$

where

$$
\begin{aligned}
& \check{X}=\left[\begin{array}{cccc}
\check{X}_{11} & \check{X}_{12} & \check{X}_{13} & \check{X}_{14} \\
\check{X}_{12}^{T} & \check{X}_{22} & \check{X}_{23} & \check{X}_{24} \\
\check{X}_{13}^{T} & \check{X}_{23}^{T} & \check{X}_{33} & \check{X}_{34} \\
\check{X}_{14}^{T} & \check{X}_{24}^{T} & \check{X}_{34}^{T} & \check{X}_{44}
\end{array}\right], \\
& \check{Y}=\left[\begin{array}{cccc}
\check{Y}_{11} & \check{Y}_{12} & \check{Y}_{13} & \check{Y}_{14} \\
\check{Y}_{12}^{T} & \check{Y}_{22} & \check{Y}_{23} & \check{Y}_{24} \\
\check{Y}_{13}^{T} & \check{Y}_{23}^{T} & \check{Y}_{33} & \check{Y}_{34} \\
\check{Y}_{14}^{T} & \check{Y}_{24}^{T} & \check{Y}_{34}^{T} & \check{Y}_{44}
\end{array}\right] \text {, } \\
& \check{X}_{5}=\left[\begin{array}{llll}
\check{X}_{15}^{T} & \check{X}_{25}^{T} & \check{X}_{35}^{T} & \check{X}_{45}^{T}
\end{array}\right] \text {, } \\
& \check{Y}_{5}=\left[\begin{array}{llll}
\check{Y}_{15}^{T} & \check{Y}_{25}^{T} & \check{Y}_{35}^{T} & \check{Y}_{45}^{T}
\end{array}\right] \text {, } \\
& \check{\Phi}_{X}=\check{X}_{5}^{T} \Pi_{1}+\Pi_{1}^{T} \check{X}_{5}, \quad \check{\Phi}_{Y}=\check{Y}_{5}^{T} \Pi_{2}+\Pi_{2}^{T} \check{Y}_{5} \text {, } \\
& \Pi_{1}=\left[\begin{array}{llll}
I_{n} & -I_{n} & 0 & 0
\end{array}\right] \text {, } \\
& \Pi_{2}=\left[\begin{array}{llll}
I_{n} & 0 & -I_{n} & 0
\end{array}\right], \quad S=R+\tau \check{X}_{55}+\eta \check{Y}_{55}, \\
& \check{A}=\left[\begin{array}{llll}
A & A_{1} & 0 & C
\end{array}\right], \\
& \check{\Phi}=\left[\begin{array}{cccc}
\check{\Phi}_{11} & P A_{1} & -A^{T} P C & 0 \\
A_{1}^{T} P & -Q_{1} & -A_{1}^{T} P C & 0 \\
-C^{T} P A & -C^{T} P A_{1} & -Q_{2} & 0 \\
0 & 0 & 0 & -R
\end{array}\right], \\
& \check{\Phi}_{11}=P A+A^{T} P+Q_{1}+Q_{2} .
\end{aligned}
$$

Proposition 4 (see [27, Theorem 1]). For given scalars $\tau>0$ and $\eta>0$, systems (1a) and (1b) are asymptotically stable, if 
the operator $\mathscr{D}$ is stable and there exist real $n \times n$ matrices $U$, $P^{T}=P, W^{T}=W, Q_{k}^{T}=Q_{k}, N_{k}^{T}=N_{k}, R_{k}^{T}=R_{k}, T_{k}^{T}=T_{k}$, $M_{k}, Z_{k}(k=1,2), E_{i}, F_{i}, H_{i}, Y_{i i}(i=1, \ldots, 5)$, and $Y_{i j}(1 \leq i<$ $j \leq 5)$ such that the following LMIs are feasible:

$$
\begin{gathered}
{\left[\begin{array}{cc}
R_{2} & Z_{2} \\
Z_{2}^{T} & T_{2}
\end{array}\right] \geq 0,} \\
Q_{k} \geq 0, \quad k=1,2, \\
W>0, \quad\left[\begin{array}{cc}
R_{1} & Z_{1} \\
Z_{1}^{T} & T_{1}
\end{array}\right]>0, \\
{\left[\begin{array}{ccc}
P & M_{1} & M_{2} \\
M_{1}^{T} & \tau^{-1} Q_{1}+N_{1} & U^{T} \\
M_{2}^{T} & \eta^{-1} Q_{2}+N_{2}
\end{array}\right]>0,} \\
\Xi:=\left[\begin{array}{ccc}
\Phi+\eta Y & \tau G_{1} & -\tau F \\
\tau G_{1}^{T} & -\tau R_{1} & -\tau Z_{1} \\
-\tau F^{T} & -\tau Z_{1}^{T} & -\tau T_{1}
\end{array}\right]<0, \\
\Omega:=\left[\begin{array}{ccc}
Y & -G_{2} & H \\
-G_{2}^{T} & R_{2} & Z_{2} \\
H^{T} & Z_{2}^{T} & T_{2}
\end{array}\right] \geq 0,
\end{gathered}
$$

where

$$
\begin{gathered}
\Phi=\left[\begin{array}{ccc}
\Phi_{11} & \cdots & \Phi_{15} \\
\cdots & \cdots & \cdots \\
\Phi_{15}^{T} & \cdots & \Phi_{55}
\end{array}\right], \quad Y=\left[\begin{array}{ccc}
Y_{11} & \cdots & Y_{15} \\
\cdots & \cdots & \cdots \\
Y_{15}^{T} & \cdots & Y_{55}
\end{array}\right], \\
F=\left[\begin{array}{c}
F_{1} \\
\vdots \\
F_{5}
\end{array}\right], \quad H=\left[\begin{array}{c}
H_{1} \\
\vdots \\
H_{5}
\end{array}\right], \\
\Phi_{11}=P A+A^{T} P+Q_{1}+Q_{2}+\tau R_{1}+\eta R_{2} \\
+M_{1}+M_{1}^{T}+M_{2}+M_{2}^{T}+F_{1}+F_{1}^{T} \\
+H_{1}+H_{1}^{T}-E_{1} A-A^{T} E_{1}^{T}, \\
\Phi_{12}=P A_{1}-M_{1}+H_{2}^{T}-A^{T} E_{2}^{T}+F_{2}^{T}-F_{1}-E_{1} A_{1}, \\
\Phi_{13}=-A^{T} P C-M_{1}^{T} C-M_{2}^{T} C-M_{2} \\
-H_{1}+H_{3}^{T}+F_{3}^{T}-A^{T} E_{3}^{T}, \\
\Phi_{14}=-A^{T} E_{4}^{T}+E_{1}+F_{4}^{T}+H_{4}^{T}+\tau Z_{1}+\eta Z_{2}, \\
\Phi_{15}=-A^{T} E_{5}^{T}+F_{5}^{T}+H_{5}^{T}-E_{1} C \\
\Phi_{22}=-Q_{1}-E_{2} A_{1}-A_{1}^{T} E_{2}^{T}-F_{2}-F_{2}^{T},
\end{gathered}
$$

$$
\begin{aligned}
& \Phi_{23}=-A_{1}^{T} P C+M_{1}^{T} C-H_{2}-F_{3}^{T}-A_{1}^{T} E_{3}^{T}, \\
& \Phi_{24}=E_{2}-A_{1}^{T} E_{4}^{T}-F_{4}^{T}, \quad \Phi_{25}=-E_{2} C-A_{1}^{T} E_{5}^{T}-F_{5}^{T} \text {, } \\
& \Phi_{33}=-Q_{2}+C^{T} M_{2}+M_{2}^{T} C-H_{3}-H_{3}^{T}, \\
& \Phi_{34}=E_{3}-H_{4}^{T}, \\
& \Phi_{35}=-E_{3} C-H_{5}^{T}, \quad \Phi_{44}=E_{4}+E_{4}^{T}+W+\tau T_{1}+\eta T_{2}, \\
& \Phi_{45}=-E_{4} C+E_{5}^{T}, \quad \Phi_{55}=-W-E_{5} C-C^{T} E_{5}^{T}, \\
& G_{1}=\left[\begin{array}{llll}
N_{1}+U^{T} & -N_{1}-U^{T} & M_{1}^{T}-M_{1}^{T} C
\end{array}\right]^{T},
\end{aligned}
$$

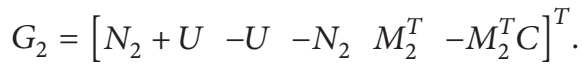

Proposition 5 (see [27, Corollary 1]). When $\eta=\tau$, for a given scalar $\tau>0$, systems (1a) and (1b) are asymptotically stable, if the operator $\mathscr{D}$ is stable and there exist real $n \times n$ matrices $W^{T}=$ $W>0, Q_{1}^{T}=Q_{1}>0, P^{T}=P, N_{1}^{T}=N_{1}, R_{1}^{T}=R_{1}, T_{1}^{T}=T_{1}$, $M_{1}, Z_{1}, E_{i}$, and $F_{i}(i=1,2,4,5)$ such that the following LMIs are feasible:

$$
\left[\begin{array}{cc}
R_{1} & Z_{1} \\
Z_{1}^{T} & T_{1}
\end{array}\right]>0, \quad\left[\begin{array}{cc}
P & M_{1} \\
M_{1}^{T} & \tau^{-1} Q_{1}+N_{1}
\end{array}\right]>0,
$$

$$
\left[\begin{array}{cccccc}
\Pi_{11} & \Pi_{12} & \Pi_{13} & \Pi_{14} & \tau N_{1} & -\tau F_{1} \\
\Pi_{12}^{T} & \Pi_{22} & \Pi_{23} & \Pi_{24} & -\tau N_{1} & -\tau F_{2} \\
\Pi_{13}^{T} & \Pi_{23}^{T} & \Pi_{33} & \Pi_{34} & \tau M_{1} & -\tau F_{4} \\
\Pi_{14}^{T} & \Pi_{24}^{T} & \Pi_{34}^{T} & \Pi_{44} & -\tau C^{T} M_{1} & -\tau F_{5} \\
\tau N_{1} & -\tau N_{1} & \tau M_{1}^{T} & -M_{1}^{T} C & -\tau R_{1} & -\tau Z_{1} \\
-\tau F_{1}^{T} & -\tau F_{2}^{T} & -\tau F_{4}^{T} & -\tau F_{5}^{T} & -\tau Z_{1}^{T} & -\tau T_{1}
\end{array}\right]<
$$

where

$$
\begin{gathered}
\Pi_{11}=P A+A^{T} P+M_{1}+M_{1}^{T}+Q_{1}+\tau R_{1} \\
+F_{1}+F_{1}^{T}-E_{1} A-A^{T} E_{1}^{T}, \\
\Pi_{12}=P A_{1}-A^{T} P C-M_{1}-M_{1}^{T} C \\
-A^{T} E_{2}^{T}-E_{1} A_{1}+F_{2}^{T}-F_{1}, \\
\Pi_{13}=-A^{T} E_{4}^{T}+E_{1}+F_{4}^{T}+\tau Z_{1}, \\
\Pi_{14}=-A^{T} E_{5}^{T}+F_{5}^{T}-E_{1} C, \\
\Pi_{22}=-Q_{1}-A_{1}^{T} P C-C^{T} P A_{1}+C^{T} M_{1}+M_{1}^{T} C \\
-E_{2} A_{1}-A_{1}^{T} E_{2}^{T}-F_{2}-F_{2}^{T}, \\
\Pi_{23}=E_{2}-A_{1}^{T} E_{4}^{T}-F_{4}^{T}, \quad \Pi_{24}=-E_{2} C-A_{1}^{T} E_{5}^{T}-F_{5}^{T}, \\
\Pi_{33}=E_{4}+E_{4}^{T}+\tau T_{1}+W, \quad \Pi_{34}=-E_{4} C+E_{5}^{T}, \\
\Pi_{44}=-E_{5} C-C^{T} E_{5}^{T}-W .
\end{gathered}
$$


Remark 6. Proposition 3 simplifies the notations in [21, Theorem 1].

Remark 7. Propositions 4 and 5 correct some slips of the pen in [27, Theorem 1 and Corollary 1]. After [27, Theorem 1] is amended as in Remark 7, [27, Theorem 2] will be correct.

\section{Simplified Stability Criteria}

In this section we will simplify the stability criteria introduced in the previous section. Firstly, the stability criterion presented in Proposition 4 can be simplified by the following theorem.

Theorem 8. For given scalars $\tau>0$ and $\eta>0$, systems (1a) and (1b) are asymptotically stable, if the operator $\mathscr{D}$ is stable and one of the following cases, (i)-(iv), holds.

(i) There exist real $n \times n$ matrices $U, P^{T}=P, W^{T}=W$, $Q_{k}^{T}=Q_{k}, N_{k}^{T}=N_{k}, R_{k}^{T}=R_{k}, T_{k}^{T}=T_{k}, M_{k}, Z_{k}(k=$ $1,2), E_{i}, F_{i}, H_{i}, Y_{i i}(i=1, \ldots, 5)$, and $Y_{i j}(1 \leq i<j \leq 5)$ such that the LMIs (10)-(15) hold.

(ii) There exist real $n \times n$ matrices $U, P^{T}=P, W^{T}=W$, $Q_{k}^{T}=Q_{k}, N_{k}^{T}=N_{k}, R_{k}^{T}=R_{k}, T_{k}^{T}=T_{k}, M_{k}, Z_{k}(k=$ $1,2), E_{i}, F_{i}, H_{i}, Y_{i i}(i=1, \ldots, 5)$, and $Y_{i j}(1 \leq i<j \leq 5)$ such that the LMIs (11)-(15) and the following LMI (20) hold:

$$
\left[\begin{array}{ll}
R_{2} & Z_{2} \\
Z_{2}^{T} & T_{2}
\end{array}\right]>0 .
$$

(iii) There exist real $n \times n$ matrices $U, P^{T}=P, W^{T}=W$, $Q_{k}^{T}=Q_{k}, N_{k}^{T}=N_{k}, R_{k}^{T}=R_{k}, T_{k}^{T}=T_{k}, M_{k}$, and $Z_{k}$ $(k=1,2)$ such that the LMIs (11), (12), and (13) and the following LMI (21) hold:

$$
\left[\begin{array}{ccccc}
\Phi & \tau G_{1} & -\tau F & -\eta G_{2} & \eta H \\
\tau G_{1}^{T} & -\tau R_{1} & -\tau Z_{1} & 0 & 0 \\
-\tau F^{T} & -\tau Z_{1}^{T} & -\tau T_{1} & 0 & 0 \\
-\eta G_{2}^{T} & 0 & 0 & -\eta R_{2} & -\eta Z_{2} \\
\eta H^{T} & 0 & 0 & -\eta Z_{2}^{T} & -\eta T_{2}
\end{array}\right]<0 .
$$

(iv) There exist real $n \times n$ matrices $U, P^{T}=P, W^{T}=W$, $Q_{k}^{T}=Q_{k}, N_{k}^{T}=N_{k}, R_{k}^{T}=R_{k}, T_{k}^{T}=T_{k}, M_{k}$, and $Z_{k}$ $(k=1,2)$ such that the LMIs (11), (12), (13), and (20) and the following LMI (22) hold:

$$
\widetilde{\Sigma}:=\left[\begin{array}{ccc}
\widetilde{\Phi} & \widetilde{G}_{1} & \widetilde{G}_{2} \\
\widetilde{G}_{1}^{T} & -\tau R_{1} & 0 \\
\widetilde{G}_{2}^{T} & 0 & -\eta R_{2}
\end{array}\right]<0,
$$

where

$$
\begin{aligned}
& \widetilde{\Phi}=\left[\begin{array}{cccc}
\widetilde{\Phi}_{11} & \widetilde{\Phi}_{12} & \widetilde{\Phi}_{13} & \widetilde{\Phi}_{14} \\
\widetilde{\Phi}_{12}^{T} & \widetilde{\Phi}_{22} & \widetilde{\Phi}_{23} & A_{1}^{T} S C \\
\widetilde{\Phi}_{13}^{T} & \widetilde{\Phi}_{23}^{T} & \widetilde{\Phi}_{33} & 0 \\
\widetilde{\Phi}_{14}^{T} & C^{T} S A_{1} & 0 & C^{T} S C-W
\end{array}\right], \\
& \widetilde{\Phi}_{11}=P A+A^{T} P+Q_{1}+Q_{2}+\tau R_{1}+\eta R_{2}+M_{1}+M_{1}^{T} \\
& +M_{2}+M_{2}^{T}+A^{T} S A+\tau A^{T} Z_{1}^{T}+\tau Z_{1} A \\
& +\eta A^{T} Z_{2}^{T}+\eta Z_{2} A-\tau^{-1} T_{1}-\eta^{-1} T_{2} \\
& \widetilde{\Phi}_{12}=P A_{1}-M_{1}+\tau Z_{1} A_{1}+\eta Z_{2} A_{1}+A^{T} S A_{1}+\tau^{-1} T_{1} \text {, } \\
& \widetilde{\Phi}_{13}=-A^{T} P C-M_{1}^{T} C-M_{2}^{T} C-M_{2}+\eta^{-1} T_{2}, \\
& \widetilde{\Phi}_{14}=\tau Z_{1} C+\eta Z_{2} C+A^{T} S C, \\
& \widetilde{\Phi}_{22}=-Q_{1}-\tau^{-1} T_{1}+A_{1}^{T} S A_{1}, \quad \widetilde{\Phi}_{23}=-A_{1}^{T} P C+M_{1}^{T} C, \\
& \widetilde{\Phi}_{33}=-Q_{2}+C^{T} M_{2}+M_{2}^{T} C-\eta^{-1} T_{2}, \\
& S=W+\tau T_{1}+\eta T_{2}, \\
& \widetilde{G}_{1}=\left[\begin{array}{c}
\tau N_{1}^{T}+\tau U-Z_{1}^{T}+\tau A^{T} M_{1} \\
-\tau N_{1}^{T}+Z_{1}^{T}+\tau A_{1}^{T} M_{1} \\
-\tau U \\
0
\end{array}\right] \text {, } \\
& \widetilde{G}_{2}=\left[\begin{array}{c}
-\eta N_{2}^{T}-\eta U^{T}+Z_{2}^{T}-\eta A^{T} M_{2} \\
\eta U^{T}-\eta A_{1}^{T} M_{2} \\
\eta N_{2}^{T}-Z_{2}^{T} \\
0
\end{array}\right] .
\end{aligned}
$$

Proof. Due to Proposition 4, it suffices to show that the conditions (i)-(iv) are equivalent.

(i) $\Rightarrow$ (ii) Since the eigenvalues of a matrix are continuous functions of its elements, it follows from (i) that there exists a sufficiently small positive scalar $\epsilon$ such that

$$
\begin{gathered}
\Xi+\operatorname{diag}(\eta \epsilon I, 0,0, \eta \epsilon I, 0,0,0)<0, \\
\Omega+\operatorname{diag}(0, \epsilon I, \epsilon I) \geq 0, \\
{\left[\begin{array}{cc}
R_{2}+\epsilon I & Z_{2} \\
Z_{2}^{T} & T_{2}+\epsilon I
\end{array}\right]>0 .}
\end{gathered}
$$

Therefore, the LMIs (11)-(15) and (20) are feasible.

(ii) $\Rightarrow$ (i) The proof is very easy, and hence it is omitted. 
(ii) $\Rightarrow$ (iii) By Lemma 1, it follows from (20) and (15) that

$$
Y \geq\left[\begin{array}{ll}
-G_{2} & H
\end{array}\right]\left[\begin{array}{cc}
R_{2} & Z_{2} \\
Z_{2}^{T} & T_{2}
\end{array}\right]^{-1}\left[\begin{array}{ll}
-G_{2} & H
\end{array}\right]^{T} .
$$

This, together with (14) and Lemma 1, implies that (21) holds. and

(iii) $\Rightarrow$ (ii) It follows from (21) and Lemma 1 that (20) holds

$$
\left[\begin{array}{ccc}
\Phi+\eta Y & \tau G_{1} & -\tau F \\
\tau G_{1}^{T} & -\tau R_{1} & -\tau Z_{1} \\
-\tau F^{T} & -\tau Z_{1}^{T} & -\tau T_{1}
\end{array}\right]<0,
$$

where

$$
Y=\left[\begin{array}{ll}
-G_{2} & H
\end{array}\right]\left[\begin{array}{cc}
R_{2} & Z_{2} \\
Z_{2}^{T} & T_{2}
\end{array}\right]^{-1}\left[\begin{array}{ll}
-G_{2} & H
\end{array}\right]^{T},
$$

and hence (ii) holds.

(iii) $\Leftrightarrow$ (iv) Let

$$
\begin{aligned}
& \widetilde{F}=\left[\begin{array}{lll}
F & H & E
\end{array}\right], \quad E=\left[\begin{array}{lllll}
E_{1}^{T} & E_{2}^{T} & E_{3}^{T} & E_{4}^{T} & E_{1}^{5}
\end{array}\right]^{T}, \\
& K=\left[\begin{array}{lllll}
e_{1}^{T} & e_{2}^{T} & e_{3}^{T} & e_{4}^{T} & e_{5}^{T}
\end{array}\right]^{T} \\
& L=\left[\begin{array}{lll}
\left(e_{1}-e_{2}-\tau e_{7}\right)^{T} & \left(e_{1}-e_{3}+\eta e_{9}\right)^{T} \widetilde{A}^{T}
\end{array}\right]^{T}, \\
& \widetilde{A}=\left[\begin{array}{llllll}
-A & -A_{1} & 0 & I & -C & 0_{n \times 4 n}
\end{array}\right], \\
& e_{i}=\left[\begin{array}{lll}
0_{n \times(i-1) n} & I & 0_{n \times(9-i) n}
\end{array}\right], \quad i=1,2, \ldots, 9 .
\end{aligned}
$$

Then LMI (21) can be written as

$$
\Phi_{1}+K^{T} \widetilde{F} L+L^{T} \widetilde{F}^{T} K<0
$$

where the matrix $\Phi_{1}$ is obtained from the matrix on the left of (21) by deleting all parts containing one of $F, H$, and $E$ (e.g., the $(5,5)$ th subblock of $\Phi_{1}$ equals $-W$, which is obtained from $\Phi_{55}$ by deleting $-E_{5} C-C^{T} E_{5}^{T}$ ). Choose

$$
\begin{aligned}
& \mathcal{N}_{K}=\left[\begin{array}{llll}
e_{6}^{T} & e_{7}^{T} & e_{8}^{T} & e_{9}^{T}
\end{array}\right], \\
& \mathscr{N}_{L}=\left[\begin{array}{c}
e_{1}+A^{T} e_{4}+\tau^{-1} e_{7}-\eta^{-1} e_{9} \\
e_{2}+A_{1}^{T} e_{4}-\tau^{-1} e_{7} \\
e_{3}+\eta^{-1} e_{9} \\
C^{T} e_{4}+e_{5} \\
e_{6} \\
e_{8}
\end{array}\right] \text {. }
\end{aligned}
$$

Noting that $\mathcal{N}_{K}^{T} \Phi_{1} \mathcal{N}_{K}<0$, one can derive from (12) and Lemma 2 that the LMI (29) (i.e., (21)) is feasible if and only if LMIs (22) and (20) are feasible.

The proof is completed.
Clearly, the stability condition (iv) in Theorem 8 is more simpler than (i) in Theorem 8 (i.e., Proposition 4). By a process similar to investigating Theorem 8 , one can easily obtain the following theorem which simplifies the stability criterion presented in Proposition 5.

Theorem 9. When $\eta=\tau$, for a given scalar $\tau>0$, systems (1a) and (1b) are asymptotically stable, if the operator $\mathscr{D}$ is stable, and one of the following cases, (i)-(ii), holds.

(i) There exist real $n \times n$ matrices $W^{T}=W>0, Q_{1}^{T}=$ $Q_{1}>0, P^{T}=P, N_{1}^{T}=N_{1}, R_{1}^{T}=R_{1}, T_{1}^{T}=T_{1}, M_{1}, Z_{1}$, $E_{i}$, and $F_{i}(i=1,2,4,5)$ such that the LMIs (17) and (18) hold.

(ii) There exist real $n \times n$ matrices $W^{T}=W>0, Q_{1}^{T}=$ $Q_{1}>0, P^{T}=P, N_{1}^{T}=N_{1}, R_{1}^{T}=R_{1}, T_{1}^{T}=T_{1}, M_{1}$, and $Z_{1}$ such that (17) and the following LMI (31) hold:

$$
\left[\begin{array}{cccc}
\widetilde{\Pi}_{11} & \widetilde{\Pi}_{12} & \widetilde{\Pi}_{13} & \widetilde{\Pi}_{14} \\
\widetilde{\Pi}_{12}^{T} & \widetilde{\Pi}_{22} & \widetilde{\Pi}_{23} & \widetilde{\Pi}_{24} \\
\widetilde{\Pi}_{13}^{T} & \widetilde{\Pi}_{23}^{T} & \widetilde{\Pi}_{33} & 0 \\
\widetilde{\Pi}_{14}^{T} & \widetilde{\Pi}_{24}^{T} & 0 & -\tau R_{1}
\end{array}\right]<0,
$$

where

$$
\begin{gathered}
\widetilde{\Pi}_{11}=P A+A^{T} P+M_{1}+M_{1}^{T}+Q_{1}+\tau R_{1} \\
+\tau Z_{1} A+\tau A^{T} Z_{1}^{T}+A^{T} S_{0} A-\tau^{-1} T_{1}, \\
\widetilde{\Pi}_{12}=P A_{1}-A^{T} P C-M_{1}-M_{1}^{T} C \\
+\tau Z_{1} A_{1}+A^{T} S_{0} A_{1}+\tau^{-1} T_{1}, \\
\widetilde{\Pi}_{13}=\tau Z_{1} C+A^{T} S_{0} C, \quad \widetilde{\Pi}_{14}=\tau N_{1}+\tau A^{T} M_{1}-Z_{1}^{T}, \\
\widetilde{\Pi}_{22}=-Q_{1}-A_{1}^{T} P C-C^{T} P A_{1}+C^{T} M_{1} \\
+M_{1}^{T} C+A_{1}^{T} S_{0} A_{1}-\tau^{-1} T_{1}, \\
\widetilde{\Pi}_{23}=A_{1}^{T} S_{0} C, \quad \widetilde{\Pi}_{24}=-\tau N_{1}+\tau A_{1}^{T} M_{1}+Z_{1}^{T}, \\
\widetilde{\Pi}_{33}=C^{T} S_{0} C-W, \quad S_{0}=W+\tau T_{1} .
\end{gathered}
$$

Remark 10. Theorems 8 and 9 indicate that, without increasing conservativeness of stability criteria, the free weighting matrices required in Propositions 4 and 5 (i.e., [27, Theorem 1 and Corollary 1]) can be eliminated. This will obviously reduce the computational complexity, and hence the stability conditions (iv) of Theorem 8 and (ii) of Theorem 9 are more effective.

Remark 11. In the special case that $M_{1}=0, N_{1}=0, Z_{1}=0$, and $R_{1}=\epsilon I_{n}$ ( $\epsilon$ is a sufficiently small positive number), Theorem 9 reduces to [26, Corollary 1]. So, by choosing suitable $M_{1}, N_{1}, Z_{1}$, and $R_{1}$, Theorem 9 could reduce the conservativeness of [26, Corollary 1$]$, by which the improvement over [26, Corollary 1] is theoretically demonstrated. 
If we choose $U=0, M_{i}=0, N_{i}=0, Z_{i}=0$, and $R_{i}=\epsilon I_{n}$ ( $\epsilon$ is a sufficiently small positive number) $(i=1,2)$ in Theorem 8 , the following corollary can be easily derived.

Corollary 12. For given scalars $\tau>0$ and $\eta>0$, systems (1a) and (1b) are asymptotically stable, if the operator $\mathscr{D}$ is stable and there exist real $n \times n$ matrices $P^{T}=P>0, W^{T}=W>0$, $Q_{k}^{T}=Q_{k}>0$, and $T_{k}^{T}=T_{k}>0(k=1,2)$ such that $L M I(33)$ holds:

$$
\widehat{\Phi}:=\left[\begin{array}{cccc}
\widehat{\Phi}_{11} & \widehat{\Phi}_{12} & \widehat{\Phi}_{13} & A^{T} S C \\
\widehat{\Phi}_{12}^{T} & \widehat{\Phi}_{22} & \widehat{\Phi}_{23} & A_{1}^{T} S C \\
\widehat{\Phi}_{13}^{T} & \widehat{\Phi}_{23}^{T} & \widehat{\Phi}_{33} & 0 \\
C^{T} S A & C^{T} S A_{1} & 0 & C^{T} S C-W
\end{array}\right]<0
$$

where

$$
\begin{gathered}
\widehat{\Phi}_{11}=P A+A^{T} P+Q_{1}+Q_{2}+A^{T} S A-\tau^{-1} T_{1}-\eta^{-1} T_{2}, \\
\widehat{\Phi}_{12}=P A_{1}+A^{T} S A_{1}+\tau^{-1} T_{1}, \quad \widehat{\Phi}_{13}=-A^{T} P C+\eta^{-1} T_{2}, \\
\widehat{\Phi}_{22}=-Q_{1}-\tau^{-1} T_{1}+A_{1}^{T} S A_{1}, \quad \widehat{\Phi}_{23}=-A_{1}^{T} P C, \\
\widehat{\Phi}_{33}=-Q_{2}-\eta^{-1} T_{2}, \quad S=W+\tau T_{1}+\eta T_{2} .
\end{gathered}
$$

Next we will show that the pair of stability criteria presented in Proposition 3 (i.e., [21, Theorem 1]) and Corollary 12 have the same conservativeness. However, the stability criterion presented in Corollary 12 is more simpler than the one in Proposition 3 (i.e., [21, Theorem 1]), which reduces greatly the computational complexity.

Theorem 13. For given scalars $\tau>0$ and $\eta>0$, LMIs (7) and (8) are feasible if and only if the LMI (33) is feasible.

Proof. The "Only If" Part. It follows from (7) and (8) that

$$
\begin{gathered}
\check{X} \geq \check{X}_{5}^{T} \check{X}_{55}^{-1} \check{X}_{5}, \quad \check{Y} \geq \check{Y}_{5}^{T} \check{Y}_{55}^{-1} \check{Y}_{5}, \\
\check{\Phi}+\tau \check{X}+\eta \check{Y}+\check{\Phi}_{X}+\check{\Phi}_{Y}+\check{A}^{T} S \check{A}<0,
\end{gathered}
$$

and hence

$$
\check{\Phi}+\tau \check{X}_{5}^{T} \check{X}_{55}^{-1} \check{X}_{5}+\eta \check{Y}_{5}^{T} \check{Y}_{55}^{-1} \check{Y}_{5}+\check{\Phi}_{X}+\check{\Phi}_{Y}+\check{A}^{T} S \check{A}<0 .
$$

Noting that

$$
\begin{gathered}
\tau \check{X}_{5}^{T} \check{X}_{55}^{-1} \check{X}_{5}+\check{\Phi}_{X}+\tau^{-1} \Pi_{1}^{T} \check{X}_{55} \Pi_{1} \geq 0, \\
\eta \check{Y}_{5}^{T} \check{Y}_{55}^{-1} \check{Y}_{5}+\check{\Phi}_{Y}+\eta^{-1} \Pi_{2}^{T} \check{Y}_{55} \Pi_{2} \geq 0,
\end{gathered}
$$

one can conclude from (36) that

$$
\check{\Phi}-\tau^{-1} \Pi_{1}^{T} \check{X}_{55} \Pi_{1}-\eta^{-1} \Pi_{2}^{T} \check{Y}_{55} \Pi_{2}+\check{A}^{T} S \check{A}<0,
$$

TABLE 1: The maximum allowable bounds of $\tau=\eta$.

\begin{tabular}{lc}
\hline Method & $\max \tau$ \\
\hline$[9]$ & 0.3 \\
{$[10]$} & 0.5658 \\
{$[11]$} & 0.5937 \\
{$[12]$} & 0.6054 \\
{$[13]$} & 0.6612 \\
{$[14]$} & 0.7039 \\
{$[15]$} & 0.74 \\
{$[16]$} & 0.8844 \\
{$[17]$} & 1.0402 \\
{$[18]$} & 1.3718 \\
{$[19]$} & 1.6014 \\
{$[20]$} & 1.61 \\
{$[21]$} & 1.6527 \\
{$[22]$} & 1.7191 \\
{$[23]$} & 1.7220 \\
{$[24]$} & 1.78 \\
{$[25]$} & 1.7844 \\
{$[26$, Corollary 1] } & 1.7856 \\
{$[27]$ and Theorem 9} & 1.7890 \\
\hline &
\end{tabular}

which becomes LMI (33) by letting $W=R, T_{1}=\check{X}_{55}$, and $T_{2}=\check{T}_{55}$. That is, LMI (33) is feasible.

The "If" Part. Since LMI (33) is feasible, there exist real $n \times n$ matrices $P^{T}=P>0, W^{T}=W>0, Q_{k}^{T}=Q_{k}>0$, and $T_{k}^{T}=T_{k}>0(k=1,2)$ such that LMI $(33)$ is satisfied. Set $R=W, \check{X}_{55}=T_{1}$, and $\check{Y}_{55}=T_{2}$. Then it follows from (33) and Lemma 1 that

$$
\left[\begin{array}{cc}
\check{\Phi}-\tau^{-1} \Pi_{1}^{T} \check{X}_{55} \Pi_{1}-\eta^{-1} \Pi_{2}^{T} \check{Y}_{55} \Pi_{2} & \check{A}^{T} S \\
S \check{A} & -S
\end{array}\right]<0 .
$$

Let

$$
\begin{aligned}
\check{X}_{5} & =-\tau^{-1} \check{X}_{55} \Pi_{1}, & \check{X} & =\check{X}_{5}^{T} \check{X}_{55}^{-1} \check{X}_{5}, \\
\check{Y}_{5} & =-\eta^{-1} \check{Y}_{55} \Pi_{2}, & \check{Y} & =\check{Y}_{5}^{T} \check{Y}_{55}^{-1} \check{Y}_{5} .
\end{aligned}
$$

Then LMI (7) holds, and

$$
\begin{gathered}
\tau \check{X}_{5}^{T} \check{X}_{55}^{-1} \check{X}_{5}+\check{\Phi}_{X}+\tau^{-1} \Pi_{1}^{T} \check{X}_{55} \Pi_{1}=0, \\
\eta \check{Y}_{5}^{T} \check{Y}_{55}^{-1} \check{Y}_{5}+\check{\Phi}_{Y}+\eta^{-1} \Pi_{2}^{T} \check{Y}_{55} \Pi_{2}=0 .
\end{gathered}
$$

Furthermore, LMI (8) is derived from (39).

The proof is completed.

\section{Numerical Comparisons of Stability Criteria}

In this section we will present one example to demonstrate effectiveness of the theoretic results described above. 
TABLE 2: The maximum allowable bounds of $\tau$ for different $\eta$.

\begin{tabular}{|c|c|c|c|c|c|c|c|c|c|c|c|c|c|c|c|c|c|c|}
\hline Method & $\eta$ & 0.1 & 0.2 & 0.3 & 0.4 & 0.5 & 0.6 & 0.7 & 0.8 & 0.9 & 1.0 & 1.1 & 1.2 & 1.3 & 1.4 & 1.5 & 10000 & 100000 \\
\hline $\begin{array}{l}{[21] \text { and }} \\
\text { Corollary } 12\end{array}$ & $\tau$ & 1.7100 & 1.6987 & 1.6883 & 1.6792 & 1.6718 & 1.6664 & 1.6624 & 1.6591 & 1.6563 & 1.6543 & 1.6531 & 1.6527 & 1.6527 & 1.6527 & 1.6527 & 1.6527 & 1.6527 \\
\hline$[25]$ & $\tau$ & 1.7844 & 1.7757 & 1.7669 & 1.7581 & 1.7495 & 1.7413 & 1.7338 & 1.7273 & 1.7226 & 1.7201 & 1.7193 & 1.7191 & 1.7191 & 1.7191 & 1.7191 & 1.7191 & 1.7191 \\
\hline $\begin{array}{l}{[27] \text { and }} \\
\text { Theorem } 8\end{array}$ & $\tau$ & 1.8305 & 1.8174 & 1.8038 & 1.7897 & 1.7755 & 1.7616 & 1.7484 & 1.7366 & 1.7272 & 1.7213 & 1.7202 & 1.7202 & 1.7202 & 1.7202 & 1.7202 & 1.7193 & 1.7193 \\
\hline
\end{tabular}

Example 1. Consider the stability of systems (1a) and (1b) with

$$
\begin{gathered}
A=\left[\begin{array}{cc}
-0.9 & 0.2 \\
0.1 & -0.9
\end{array}\right], \quad A_{1}=\left[\begin{array}{ll}
-1.1 & -0.2 \\
-0.1 & -1.1
\end{array}\right], \\
C=\left[\begin{array}{cc}
-0.2 & 0 \\
0.2 & -0.1
\end{array}\right] .
\end{gathered}
$$

Case $1(\tau=\eta)$. The upper bounds on the delay $\tau$ under which robust stability of this system can be guaranteed using the methods in [9-27] and Theorem 9 of this paper are listed in Table 1. It is clear that our results are significantly better than those in [9-26] because our allowable maximum time delay $\tau$ is larger.

Case $2(\tau \neq \eta)$. For different values of $\eta$, Table 2 lists the maximum allowable bounds on $\tau$ that guarantee the stability of the system. It can be seen that the maximum allowable bound on $\tau$ decreases as $\eta$ increases when $\eta$ is small but that $\tau$ remains almost unchanged when $\eta$ increases to certain extent.

\section{Conclusions}

By using matrix analysis techniques, several stability criteria (i.e., [21, Theorem 1] and [27, Theorem 1 and Corollary 1]) for delayed neutral systems have been simplified. The numbers of LMI variables in [21, Theorem 1] and [27, Theorem 1 and Corollary 1] are reduced into the simplified ones, which can obviously reduce computational complexity. Furthermore, it is theoretically proven that the simplified stability criteria have the same conservativeness as the original ones. A numerical example is given to illustrate the theoretic results investigated in this paper.

Extending the idea of this paper to other system models, including singular delayed systems [32-35], stochastic systems [36], Markovian jump systems [37, 38], and genetic regulatory networks [39-41], is under consideration.

\section{Conflict of Interests}

The authors declare that there is no commercial or associative interest that represents a conflict of interest in connection with the work submitted.

\section{Acknowledgments}

This work is supported by the National Natural Science Foundation of China under Grant no. 11371006, the fund of Heilongjiang Province Innovation Team Support Plan under
Grant no. 2012TD007, the fund of Heilongjiang University Innovation Team Support Plan under Grant no. Hdtd201003, the Fund of Key Laboratory of Electronics Engineering, College of Heilongjiang Province, (Heilongjiang University), China, and the fund of Heilongjiang Education Committee. The authors thank the anonymous referees for their helpful comments and suggestions which improve greatly this paper.

\section{References}

[1] V. B. Kolmanovskii and A. Myshkis, Applied Theory of Functional Differential Equations, Kluwer Academic Publishers, Boston, Mass, USA, 1992.

[2] Y. Kuang, Delay Differential Equations with Applications in Population Dynamics, vol. 191 of Mathematics in Science and Engineering, Academic Press, Boston, Mass, USA, 1993.

[3] S.-I. Niculescu, Delay Effects on Stability, vol. 269 of Lecture Notes in Control and Information Sciences, Springer, Berlin, Germany, 2001.

[4] X. Lin, X. Zhang, and Y. Wang, "Robust passive filtering for neutral-type neural networks with time-varying discrete and unbounded distributed delays," Journal of the Franklin Institute, vol. 350, no. 5, pp. 966-989, 2013.

[5] X. Zhang, X. Lin, and Y. Wang, "Robust fault detection filter design for a class of neutral-type neural networks with timevarying discrete and unbounded distributed delays," Optimal Control Applications \& Methods, vol. 34, no. 5, pp. 590-607, 2013.

[6] Y. Wang, X. Zhang, and Y. Hu, "Robust $H_{\infty}$ control for a class of uncertain neutral stochastic systems with mixed delays: A CCL approach," Circuits, Systems, and Signal Processing, vol. 32, no. 2, pp. 631-646, 2013.

[7] Y. Wang, X. Zhang, and Y. He, "Improved delay-dependent robust stability criteria for a class of uncertain mixed neutral and Lur'e dynamical systems with interval time-varying delays and sector-bounded nonlinearity," Nonlinear Analysis: Real World Applications, vol. 13, no. 5, pp. 2188-2194, 2012.

[8] G. D. Zhang, X. Lin, and X. Zhang, "Exponential stabilization of neutral-type neural networks with mixed interval time-varying delays by intermittent control: a CCL approach," Circuits, Systems and Signal Processing, vol. 33, pp. 371-391, 2014.

[9] C.-H. Lien, K.-W. Yu, and J.-G. Hsieh, "Stability conditions for a class of neutral systems with multiple time delays," Journal of Mathematical Analysis and Applications, vol. 245, no. 1, pp. 2027, 2000.

[10] J. D. Chen, C. H. Lien, K. K. Fan, and J. H. Chou, "Criteria for asymptotic stability of a class of neutral systems via a LMI approach," IEE Proceedings: Control Theory and Applications, vol. 148, no. 6, pp. 442-447, 2001.

[11] Y. He, Q.-G. Wang, C. Lin, and M. Wu, "Augmented Lyapunov functional and delay-dependent stability criteria for neutral systems," International Journal of Robust and Nonlinear Control, vol. 15, no. 18, pp. 923-933, 2005. 
[12] M. Wu, Y. He, and J.-H. She, "New delay-dependent stability criteria and stabilizing method for neutral systems," IEEE Transactions on Automatic Control, vol. 49, no. 12, pp. 22662271, 2004.

[13] J. Sun, G. P. Liu, and J. Chen, "Delay-dependent stability and stabilization of neutral time-delay systems," International Journal of Robust and Nonlinear Control, vol. 19, no. 12, pp. 13641375, 2009.

[14] W. Qian, J. Liu, and S. Fei, "Augmented Lyapunov functional approach for stability of neutral systems with mixed delays," Asian Journal of Control, vol. 14, no. 2, pp. 572-579, 2012.

[15] E. Fridman, "New Lyapunov-Krasovskii functionals for stability of linear retarded and neutral type systems," Systems \& Control Letters, vol. 43, no. 4, pp. 309-319, 2001.

[16] C.-H. Lien and J.-D. Chen, "Discrete-delay-independent and discrete-delay-dependent criteria for a class of neutral systems," Journal of Dynamic Systems, Measurement and Control, Transactions of the ASME, vol. 125, no. 1, pp. 33-41, 2003.

[17] H. Chen, Y. Zhang, and Y. Zhao, "Stability analysis for uncertain neutral systems with discrete and distributed delays," Applied Mathematics and Computation, vol. 218, no. 23, pp. 11351-11361, 2012.

[18] J. H. Park and O. Kwon, "On new stability criterion for delaydifferential systems of neutral type," Applied Mathematics and Computation, vol. 162, no. 2, pp. 627-637, 2005.

[19] Q.-L. Han, "On robust stability of neutral systems with timevarying discrete delay and norm-bounded uncertainty," Automatica, vol. 40, no. 6, pp. 1087-1092, 2004.

[20] Q.-L. Han, "A descriptor system approach to robust stability of uncertain neutral systems with discrete and distributed delays," Automatica, vol. 40, no. 10, pp. 1791-1796, 2004.

[21] Y. He, M. Wu, J.-H. She, and G.-P. Liu, "Delay-dependent robust stability criteria for uncertain neutral systems with mixed delays," Systems \& Control Letters, vol. 51, no. 1, pp. 57-65, 2004.

[22] O. M. Kwon, J. H. Park, and S. M. Lee, "On stability criteria for uncertain delay-differential systems of neutral type with timevarying delays," Applied Mathematics and Computation, vol. 197, no. 2, pp. 864-873, 2008.

[23] S. Xu, J. Lam, and Y. Zou, "Further results on delay-dependent robust stability conditions of uncertain neutral systems," International Journal of Robust and Nonlinear Control, vol. 15, no. 5, pp. 233-246, 2005.

[24] E. Fridman and U. Shaked, "A descriptor system approach to $H_{\infty}$ control of linear time-delay systems," IEEE Transactions on Automatic Control, vol. 47, no. 2, pp. 253-270, 2002.

[25] X.-G. Liu, M. Wu, R. Martin, and M.-L. Tang, "Stability analysis for neutral systems with mixed delays," Journal of Computational and Applied Mathematics, vol. 202, no. 2, pp. 478-497, 2007.

[26] Z. Zhao, W. Wang, and B. Yang, "Delay and its time-derivative dependent robust stability of neutral control system," Applied Mathematics and Computation, vol. 187, no. 2, pp. 1326-1332, 2007.

[27] W. Qian, J. Liu, Y. Sun, and S. Fei, "A less conservative robust stability criteria for uncertain neutral systems with mixed delays," Mathematics and Computers in Simulation, vol. 80, no. 5, pp. 1007-1017, 2010.

[28] K. Gu and S.-I. Niculescu, "Further remarks on additional dynamics in various model transformations of linear delay systems," IEEE Transactions on Automatic Control, vol. 46, no. 3, pp. 497-500, 2001.
[29] J. K. Hale and S. M. Verduyn Lunel, Introduction to FunctionalDifferential Equations, vol. 99 of Applied Mathematical Sciences, Springer, New York, NY, USA, 1993.

[30] S. Boyd, L. El Ghaoui, E. Feron, and V. Balakrishnan, Linear Matrix Inequalities in System and Control Theory, vol. 15 of SIAM Studies in Applied Mathematics, Society for Industrial and Applied Mathematics, Philadelphia, Pa, USA, 1994.

[31] P. Gahinet and P. Apkarian, "A linear matrix inequality approach to $H_{\infty}$ control," International Journal of Robust and Nonlinear Control, vol. 4, no. 4, pp. 421-448, 1994.

[32] H. Zhu, X. Zhang, and S. Cui, "Further results on $H_{\infty}$ control for discrete-time uncertain singular systems with interval timevarying delays in state and input," Optimal Control Applications \& Methods, vol. 34, no. 3, pp. 328-347, 2013.

[33] F. Li and X. Zhang, "A delay-dependent bounded real lemma for singular LPV systems with time-variant delay," International Journal of Robust and Nonlinear Control, vol. 22, no. 5, pp. 559574, 2012.

[34] F. B. Li, P. Shi, L. G. Wu, and X. Zhang, "Fuzzy-model-based D-stability and non-fragile control for discrete-time descriptor systems with multiple delays," IEEE Transactions on Fuzzy Systems, 2013.

[35] X. Zhang and H. Zhu, "Robust stability and stabilization criteria for discrete singular time-delay LPV systems," Asian Journal of Control, vol. 14, no. 4, pp. 1084-1094, 2012.

[36] L. G. Wu, W. X. Zheng, and H. J. Gao, "Dissipativity-based sliding mode control of switched stochastic systems," IEEE Transactions on Automatic Control, vol. 58, no. 3, pp. 785-791, 2013.

[37] L. Wu, X. Su, and P. Shi, "Sliding mode control with bounded $L_{2}$ gain performance of Markovian jump singular time-delay systems," Automatica, vol. 48, no. 8, pp. 1929-1933, 2012.

[38] F. B. Li, L. G. Wu, and P. Shi, "Stochastic stability of semiMarkovian jump systems with mode-dependent delays," International Journal of Robust and Nonlinear Control, 2013.

[39] Y. T. Wang, A. H. Yu, and X. Zhang, "Robust stability of stochastic genetic regulatory networks with time-varying delays: a delay fractioning approach," Neural Computing and Applications, vol. 23, no. 5, pp. 1217-1227, 2013.

[40] L. L. Chen, Y. Zhou, and X. Zhang, "Guaranteed cost control for uncertain genetic regulatory networks with interval timevarying delays," Neurocomputing, vol. 131, pp. 105-112, 2014.

[41] X. Zhang, A. H. Yu, and G. D. Zhang, "M-matrix-based delay-range-dependent global asymptotical stability criterion for genetic regulatory networks with time-varying delays," Neurocomputing, vol. 113, pp. 8-15, 2013. 


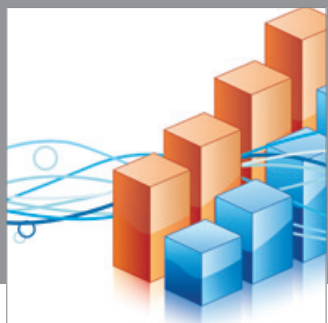

Advances in

Operations Research

mansans

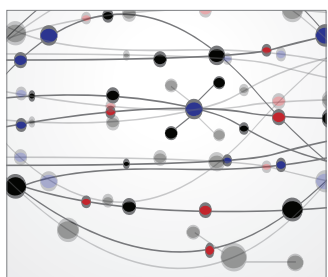

The Scientific World Journal
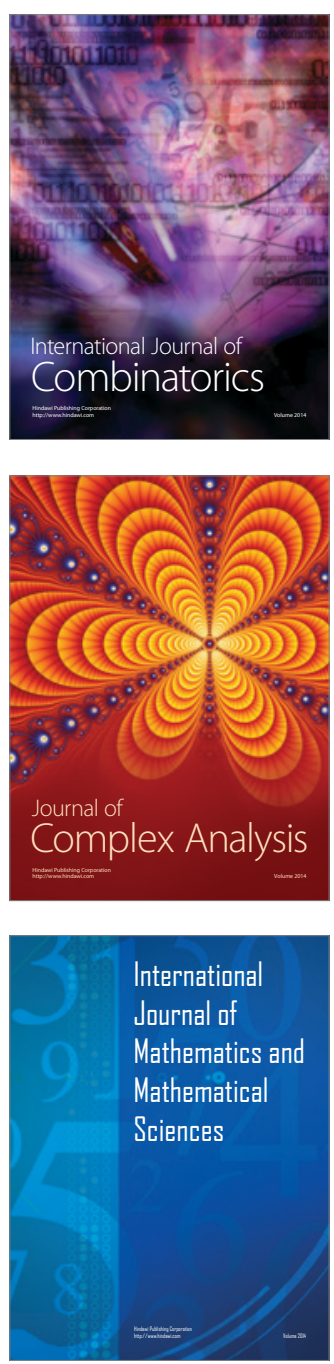
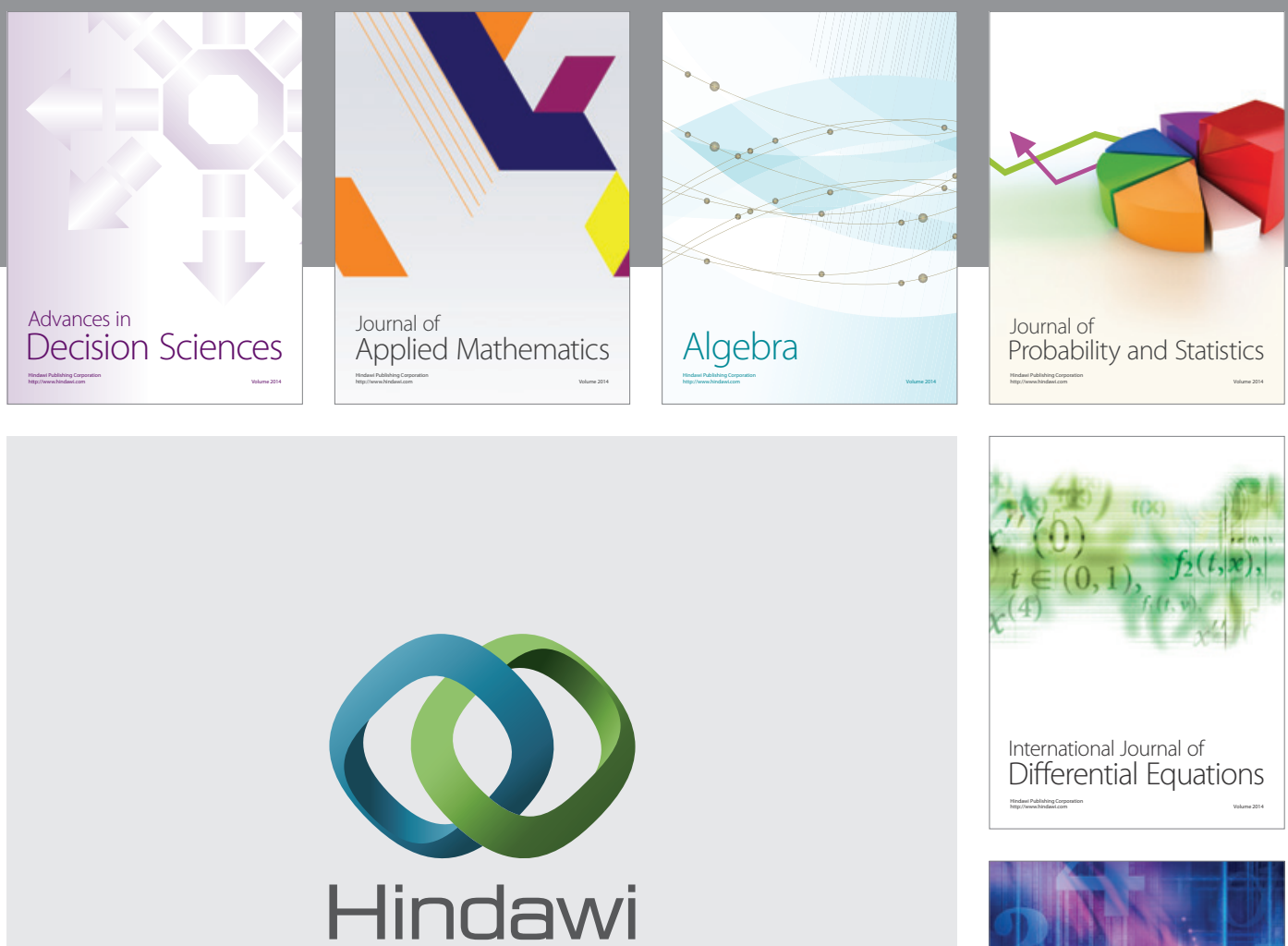

Submit your manuscripts at http://www.hindawi.com
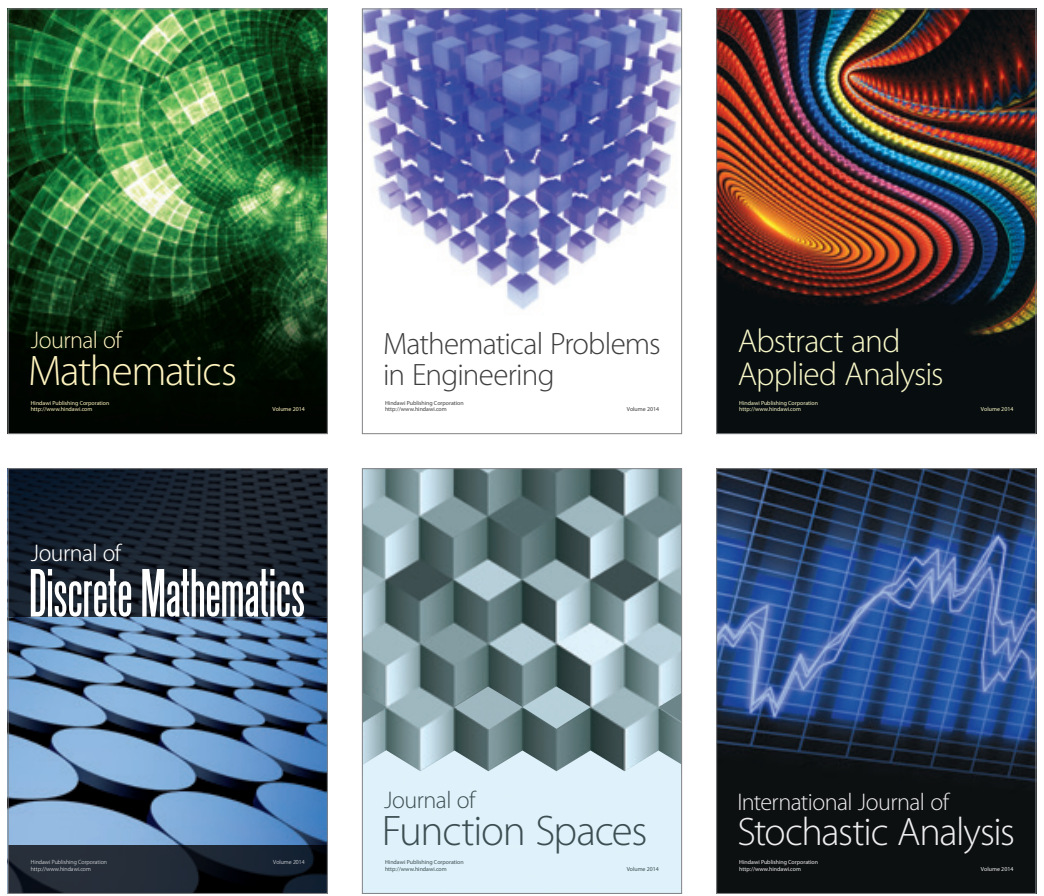

Journal of

Function Spaces

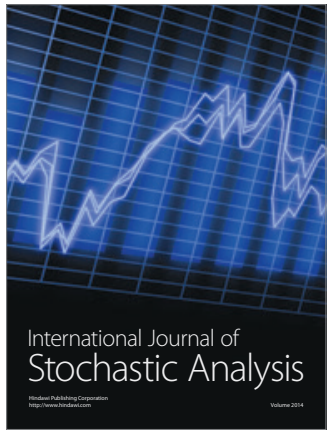

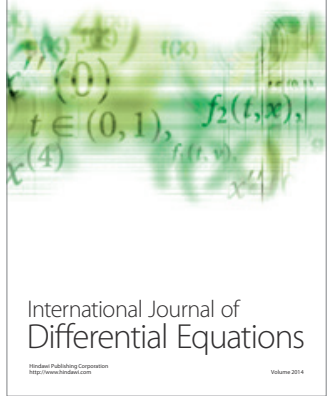
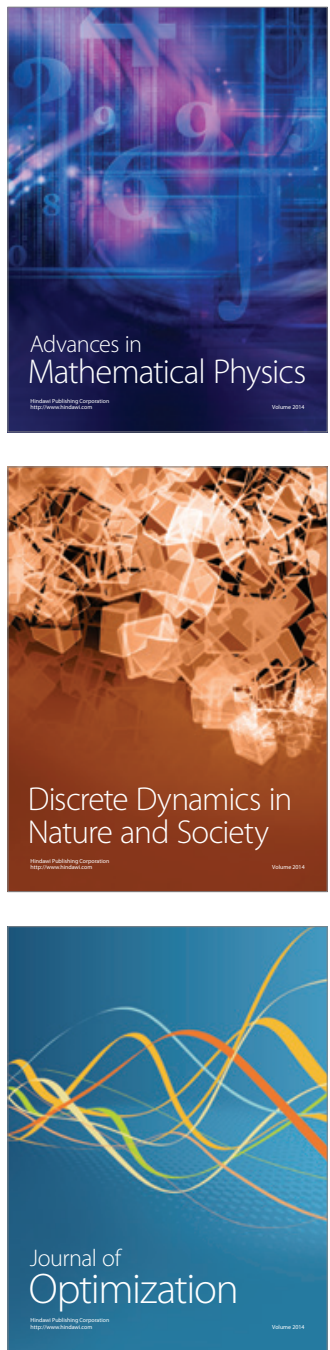